EPJ Web of Conferences 60, 12016 (2013)

DOI: $10.1051 /$ epjconf $/ 20136012016$

(C) Owned by the authors, published by EDP Sciences, 2013

\title{
Searches for exotic and rare higgs decays in CMS
}

\author{
Federica Primavera ${ }^{1,2, a}$ \\ ${ }^{1}$ University of Bologna, Italy \\ ${ }^{2}$ INFN, Bologna, Italy
}

\begin{abstract}
The results on searches for Higgs-like particles beyond the Standard Model and rare Standard Model Higgs decays are presented. Searches are performed using the data collected by CMS experiment in protonproton collision at LHC. Most recent results concerning light Higgses as predicted in the MSSM, NMSSM and fermiophobic models are discussed. Decays of the $125 \mathrm{GeV}$ Higgs-like particle to $\mathrm{Z} \gamma$ channel is also presented.
\end{abstract}

\section{Introduction}

The electroweak symmetry breaking mechanism of the Standard Model (SM) predicts the existence of a neutral scalar boson, the Higgs particle. While a boson, consistent so far with its expected properties, has been recently observed at a mass of about $125 \mathrm{GeV} / \mathrm{c}^{2}[1,2]$, its exact properties and the detailed structure of the Higgs sector still need further investigation.

The SM Higgs boson suffers from quadratically divergent self-energy corrections at high energy, and the problem can be solved only extending the model.

Indirect searches for the presence of beyond SM (BSM) particles, performed on the data collected by CMS [3], allow us to conclude that the $\mathrm{BR}_{\mathrm{BSM}}$ is in the interval $[0.00,0.52]$ at $95 \% \mathrm{CL}$, but there are no indications about the energy scale where it can occur [4].

Several extensions of the SM have been proposed and are currently studied. The simplest is the minimal supersymmetric model (MSSM), where the Higgs sector contains two Higgs boson doublets [6], one couples to the uptype and the other to the down-type fermions. After electroweak symmetry breaking, five Higgs bosons remain: the CP-odd neutral scalar $\mathrm{A}^{0}$, the two charged scalars $\mathrm{H}^{+}$ and $\mathrm{H}^{-}$, and the two CP-even neutral scalars $\mathrm{h}$ and $\mathrm{H}^{0}$. This model, containing a large number of free parameters, by some constraints, can be described in term of just two of them: the mass of the neutral scalar $\mathrm{m}_{A^{0}}$, and the ratio between the vacuum expectation values of the two doublets $\tan \beta$.

Another model is the Next-to-MSSM (NMSSM), which extends the MSSM, to resolve the problem of $\mu$ term [12], by introducing a complex scalar singlet. It expands the Higgs sector to three CP-even scalars $h_{1}, h_{2}$ and $\mathrm{h}_{3}$, two CP-odd $\mathrm{a}_{1}$ and $\mathrm{a}_{2}$, and two charged scalars $\mathrm{H}^{+}$and $\mathrm{H}^{-}$.

\footnotetext{
a e-mail: federica.primavera@cern.ch
}

Some models also predict that the Higgs couples only to vector bosons at tree level, usually referred to as fermiophobic (FP).

In addition to direct searches for BSM Higgses, searches for rare SM Higgs decays, like the Higgs in $\mathrm{Z} \gamma$, are sensitive to BSM physics and could be modified by new charged particles, by an extended Higgs sector, or by a presence of new scalars.

\section{MSSM Higgs searches}

All the MSSM studies are performed in the $\mathrm{m}_{h}^{\max }$ scenario, where constraints are set to obtain a most conservative model, in which the mass of light boson $\mathrm{h}$ is attended to be very close to the SM Higgs mass.

The searches for charged Higgs have been performed under the light mass hypothesis [7]. If $\mathrm{m}_{\mathrm{H}^{+}}<\mathrm{m}_{\mathrm{t}}-\mathrm{m}_{\mathrm{b}}$, the Higgs can be produced in top quark decays $\mathrm{t} \rightarrow \mathrm{H}+\mathrm{b}$. For values of $\tan \beta>5$, the charged Higgs boson preferentially decays to a $\tau$ lepton and a neutrino, therefore, deriving the experimental limits, we assume that the branching ratio $\mathrm{BR}\left(\mathrm{H}^{+} \rightarrow \tau^{+} v_{\tau}\right)$ is equal to 1 .

The dominant top quarks production process at LHC is $\mathrm{pp} \rightarrow \mathrm{tt}+\mathrm{X}$ via gluon fusion.

The possible decays of the top pairs are $\mathrm{tt} \rightarrow \mathrm{H}^{ \pm} \mathrm{W}^{\mp} \mathrm{b} \overline{\mathrm{b}}$ and $\mathrm{tt} \rightarrow \mathrm{H}^{ \pm} \mathrm{H}^{\mp} \mathrm{b} \overline{\mathrm{b}}$, where each charged Higgs boson decays into a $\tau$ lepton and a neutrino. Depending on the $\tau$ decay, three not-overlapped final states are searched for, all requiring missing transverse energy and multiple jets coming from the hadronization of b-quarks: fully hadronic, semi-leptonic and leptonic channel.

Depending on the final states, we have different background sources, estimated from both data and simulation.

An amount of data corresponding to $1.99-2.27 \mathrm{fb}^{-1}$, depending on the final state, recorded by CMS in pp collision at $\sqrt{s}=7 \mathrm{TeV}$, is analysed. Since no deviation is observed, upper limit on the branching ratio $\mathrm{BR}(\mathrm{t} \rightarrow \mathrm{H}+\mathrm{b})$ in the range of $2-4 \%$ are established (fig. 1), and exclusion 


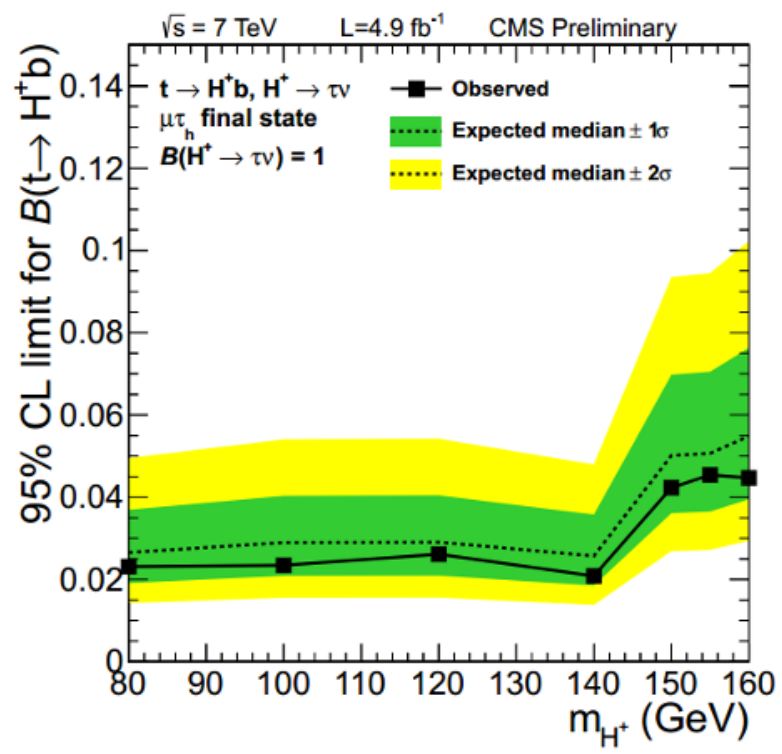

Figure 1. Upper limit for the MSSM cross-section production of $t \rightarrow \mathrm{H}^{+}+\mathrm{b}$

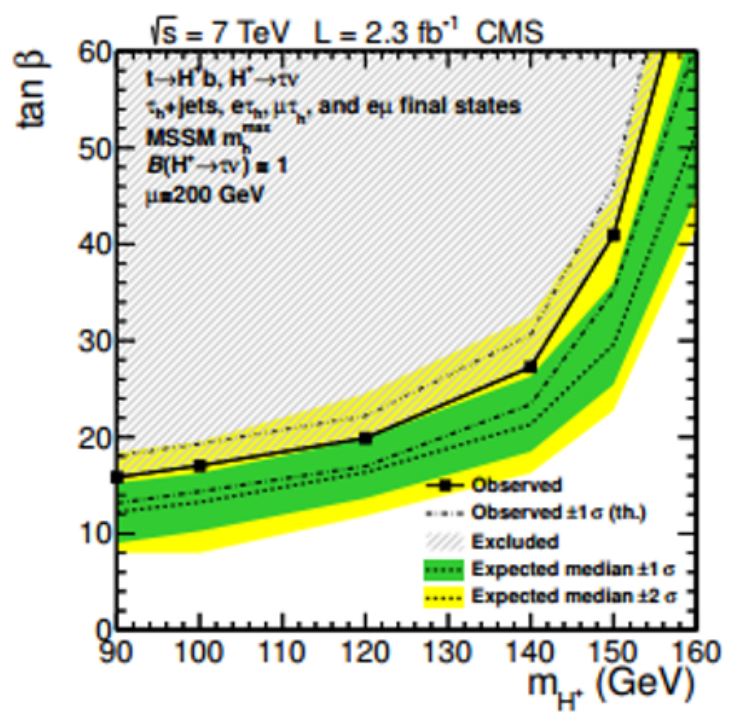

Figure 2. Exclusion region in the MSSM $\left(\mathrm{m}_{\mathrm{H}^{+}}, \tan \beta\right)$ parameter space.

region in the $\left(\mathrm{m}_{\mathrm{H}^{+}}, \tan \beta\right)$ plane (fig. 2) are set, combining the results from the three different final states.

The MSSM neutral Higgs production $\mathrm{pp} \rightarrow \Phi^{0}+\mathrm{X}$ at the LHC is dominated by two processes: $\mathrm{b} \bar{b}$-associated production, where $\Phi^{0}$ is produced together with a $b \bar{b}$ pair, and the gluon-gluon (gg) fusion process. For relatively large values of $\tan \beta$, the Higgs couplings to u-type particles are suppressed while the couplings to d-type particles are enhanced by a factor $\tan \beta$, relative to the SM. Therefore, in the MSSM, the combined cross section of Higgs boson production in association with $\mathrm{b}$ quarks is enhanced by a factor $2 \tan ^{2} \beta$.

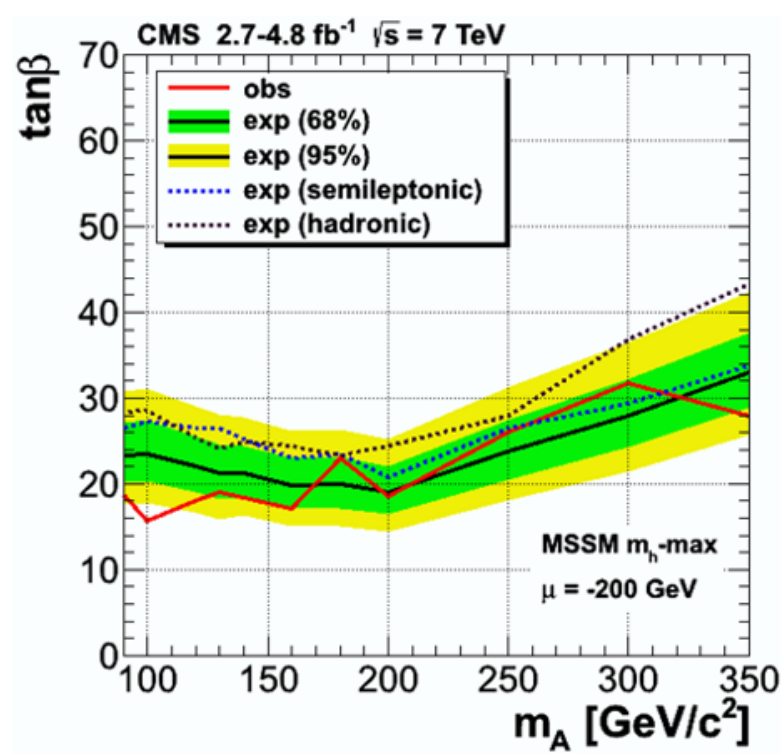

Figure 3. Exclusion limit for the MSSM $\Phi^{0} \rightarrow b \bar{b}$ in the $\left(\mathrm{m}_{A}-\right.$ $\tan \beta$ ) plane.

For the same reason the neutral Higgs decay to $b$ quarks has the highest branching fraction, $90 \%$, with the disadvantage to be difficult to separate from the very large QCD background.

Despite their low branching ratios, the $\Phi^{0} \rightarrow \tau^{+} \tau^{-}$and the $\Phi^{0} \rightarrow \mu^{+} \mu^{-}$decay channels provide higher sensitivity than $\Phi^{0} \rightarrow b \bar{b}$.

Among them, while the first process has a branching ratio larger by a factor $\left(\mathrm{m}_{\tau} / \mathrm{m}_{\mu}\right)^{2}$ and provides better sensitivity in terms of limits calculation, the $\Phi^{0} \rightarrow \mu^{+} \mu^{-}$has a cleaner experimental signature and benefits from the full reconstruction of the final state.

For the $\mathrm{b} \overline{\mathrm{b}}$ final state, in order to discriminate the signal, only the associated production is considered. Two analyses are performed for this channel: one using a fully hadronic trigger based on an high transverse momentum $\left(\mathrm{p}_{\mathrm{T}}\right)$ threshold for jets and on-line b-tagging [8], and one exploiting dedicated triggers based on a moderate $\mathrm{p}_{\mathrm{T}}$ threshold with on-line b-tagging for jets and non-isolated muons [9]. The use of a muon- and jet-triggered dataset allows to tag the semi-muonic decay of one of the b-quarks, and also to tolerate lower energy thresholds on jets in the trigger, improving the overall sensitivity, especially in the low mass region.

The main background arises from the heavy flavor multijet QCD. Both the analyses adopt a data-driven approach for the background estimation.

An amount of data corresponding to 4.0 and $4.8 \mathrm{fb}^{-1}$ for the first and second analyses respectively, recorded by CMS in pp collision at $\sqrt{s}=7 \mathrm{TeV}$, is analysed. Combining the results exclusion limit in the $\left(\mathrm{m}_{\mathrm{A}}, \tan \beta\right)$ plane is set (fig. 3).

In case of $\Phi^{0} \rightarrow \tau^{+} \tau^{-}$or $\mu^{+} \mu^{-}$decays both bassociated and gluon fusion production are taken into account. In order to maximize the sensitivity, both the analyses have a categorization based on the presence or absence 


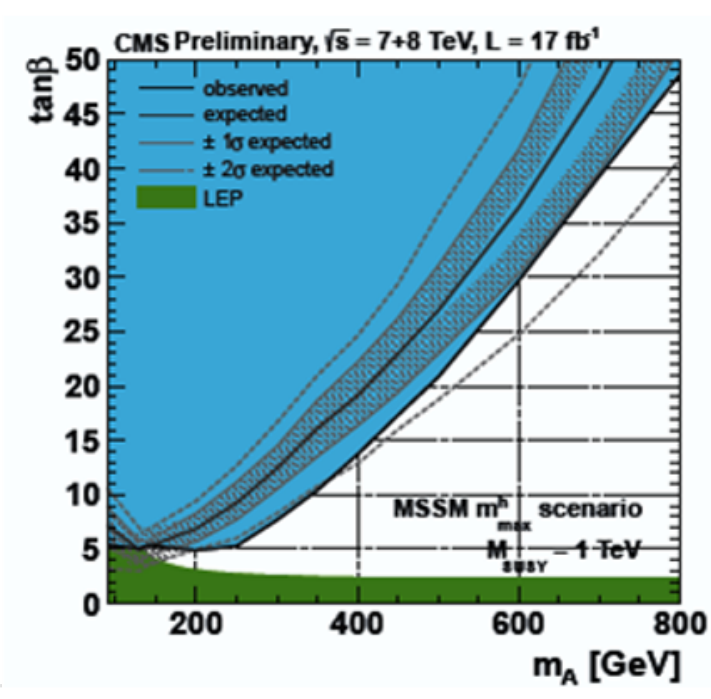

Figure 4. Exclusion limit for the MSSM $\Phi^{0} \rightarrow \tau^{+} \tau^{-}$in the $\left(\mathrm{m}_{A}, \tan \beta\right)$ plane.

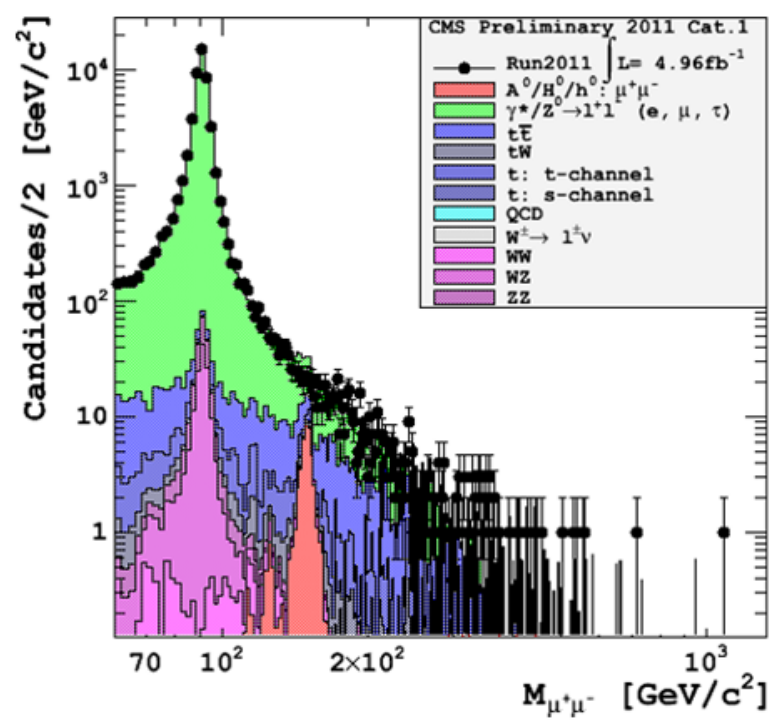

Figure 5. Invariant di-muon mass distribution for $5 \mathrm{fb}^{-1}$ of data collected at $\sqrt{s}=7 \mathrm{TeV}$. Note that simulated signal peaks, in pink, for $\mathrm{h}$ (left) and $\mathrm{A}+\mathrm{H}$ (right) can be distinguished.

of b-jets in the events. Just for $\tau^{+} \tau^{-}$channel a further categorization, based on the $\tau$ decay modes, is performed.

Plot in fig. 4 shows the exclusion limit in the $\left(\mathrm{m}_{\mathrm{A}}, \tan \beta\right)$ plane for the combination of data taken at $\sqrt{s}=7$ and 8 $\mathrm{TeV}$, for a total amount of $17 \mathrm{fb}^{-1}$. This result [10] is more restrictive than the one from the search for $\mu^{+} \mu^{-}$Higgs decay [11]. Nevertheless, this channel allows to reconstruct the Higgs mass with a better resolution, thanks to the high resolution on muon momentum. It is shown in the plot of invariant di-muon mass distribution in fig. 5 .

In case of MSSM Higgs existence the $\mu \mu$ channel makes it possible to measure the $\tan \beta$ parameter.

\section{NMSSM Higgs searches}

Searches for a new light bosons, predicted by NMSSM (and not only) and decaying in two muons, are presented for two different analysis. One searches for a prompt $\mathrm{a} \rightarrow$ $\mu^{+} \mu^{-}$in the mass range between 5.5 and $14 \mathrm{GeV}$, using 1.3 $\mathrm{fb}^{-1}$, collected in pp collision at $\sqrt{s}=7 \mathrm{TeV}$ [13]. The other searches for $\mathrm{h} \rightarrow 2 \mathrm{a}+\mathrm{X} \rightarrow 4 \mu+\mathrm{X}$ in a further low mass range between 0.25 and $3.55 \mathrm{GeV}$, using $5.3 \mathrm{fb}^{-1}$ at $\sqrt{s}=$ $7 \mathrm{TeV}$ [14].

Both require isolated muons with a specific $\mathrm{p}_{\mathrm{T}}$ cut and $\eta$ restriction, depending on the mass range.

The estimation of background is data driven for the two analyses: in the first it is constituted by QCD processes and, because we are close to mass region of the Yresonance, by the its tails; and in the second by the prompt bb pair and $\mathrm{J} / \Psi$ that decay in muons.

No Higgs is observed and upper limits for cross section times branching ratio of both processes are set.

\section{Fermiophobic Higgs searches}

Several extension of SM, where a Higgs boson couples only to the vector bosons at tree level, are predicted. Consequently, searches for FP Higgs are possible only via vector boson fusion (VBF) production, or in associated vector boson (VH) production.

Fermiophobic Higgs decaying to two photons [15] is searched in data collected at $\sqrt{s}=7$ and $8 \mathrm{TeV}$, which correspond to a total integrated luminosity of $10.4 \mathrm{fb}^{-1}$. The mass range explored is $110-150 \mathrm{GeV}$.

The data are preselected by a di-photon trigger based on a loose isolation, $\mathrm{p}_{\mathrm{T}}$ and $\eta$ requirements. At off-line level a tighter version of previous criteria is applied. Then the events are split into different categories according to the production processes.

Main background sources come from the misidentified electrons and from the photons within the jets. They are estimated with a data-driven approach.

No signal is observed and upper limit on fermiophobic cross section $\sigma(\mathrm{H} \rightarrow \gamma \gamma) / \sigma(\mathrm{H} \rightarrow \gamma \gamma)_{\mathrm{FP}}$ is set at 95\% CL. in the mass range $110-147 \mathrm{GeV}$ and at $99 \% \mathrm{CL}$. in the mass range $110-134 \mathrm{GeV}$ (fig. 6).

\section{Rare Higgs decays}

Search for the Higgs boson in the $\mathrm{Z} \gamma$ decay, where $\mathrm{Z}$ final state is one electron or muon pair [16], is presented. This search is sensitive to BSM physics because the Higgs partial width is induced by loops of heavy charged particles, provided by extensions of SM.

The analysis is performed on $10.2 \mathrm{fb}^{-1}$ of data collected at $\sqrt{s}=7$ and $8 \mathrm{TeV}$.

Events are selected if they have isolated opposite-sign same-flavour leptons consistent with a $\mathrm{Z}$ boson, and one isolated photon. The sensitivity is enhanced by dividing the events in classes according to indicators of expected Higgs mass resolution and signal to background ratio. Main sources of irreducible background come, for 


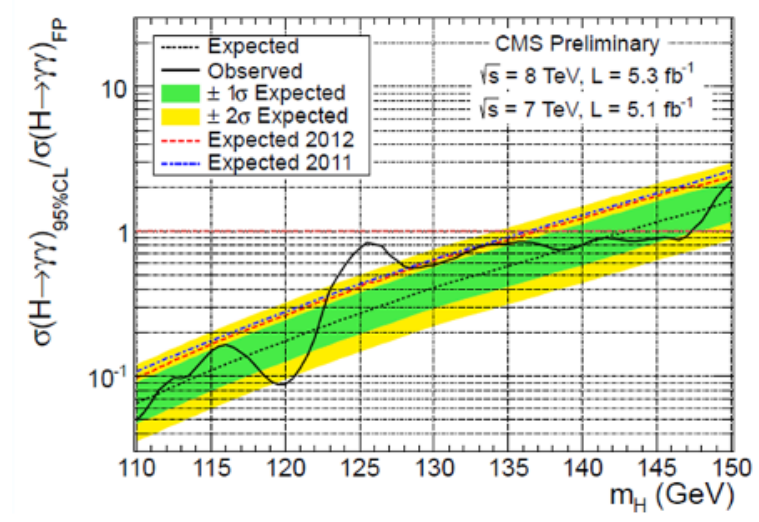

Figure 6. Upper limit on the signal strength, $\sigma / \sigma_{F P}$ of a fermiophobic Higgs.

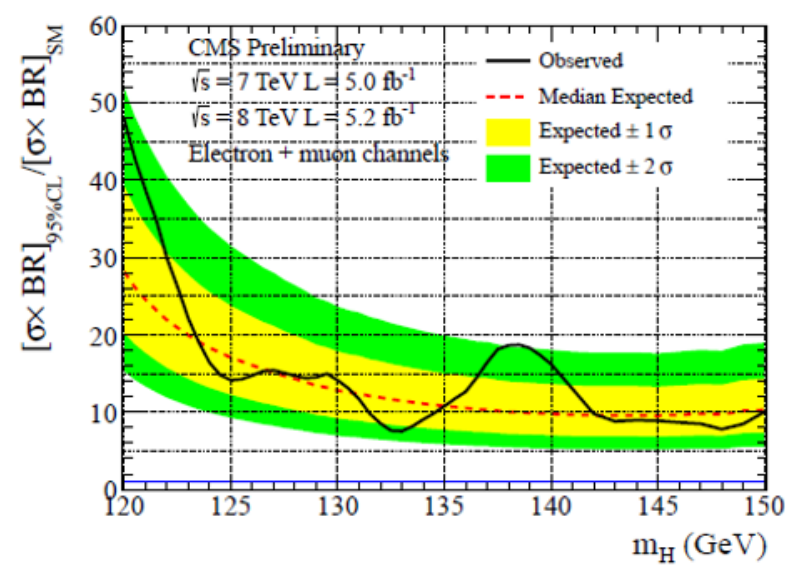

Figure 7. Exclusion limit on $\sigma(\mathrm{pp} \rightarrow \mathrm{H}) \times \mathrm{BR}(\mathrm{H} \rightarrow \mathrm{Z} \gamma)$ of $\mathrm{SM}$ Higgs.

$70 \%$, from prompt $\mathrm{pp} \rightarrow \mathrm{Z} \gamma$ production, and, for remaining $30 \%$, from Drell-Yan processes. The background shape is derived from data fitting the mass distribution of di-lepton$\gamma$.

Since no evidence of Higgs is observed, upper limit on cross section times branching ratio is derived in the mass range between 120 and $150 \mathrm{GeV}$ (fig. 7).

\section{Conclusions}

Data collected at CMS in collision at $\sqrt{s}=7$ and $8 \mathrm{TeV}$, used for indirect searches for BSM particles, indicate a non zero $\mathrm{BR}_{\mathrm{BSM}}$, but without indications about the energy scale at which it could be manifest.

Search for Higgs bosons predicted in several extensions of the SM, like MSSM, NMSSM and FP, are discussed in this letter. SM Higgs rare decays are also considered, since are sensitive to the existence of BSM particles.

No signal is observed in search for exotic and rare Higgs decays, but increasingly strict exclusion limits and upper limits are set in the Higgs parameters space and on cross section production respectively.

\section{References}

[1] CMS Collaboration: Observation of a new boson at mass of $125 \mathrm{GeV}$ with CMS experiment at LHC, Phys. Lett. B 716 (2012) 30-61.

[2] Atlas Collaboration: Observation of a new particle in the search for the Standard Model Higgs boson with the ATLAS detector at LHC, Phys. Lett. B 716 (2012) 1-29.

[3] CMS Collaboration: The CMS experiment at the CERN LHC, JINST 3:S08004 (2008).

[4] CMS Collaboration: Measurements of the properties of the new boson with a mass near to $125 \mathrm{GeV}$, CMS PAS HIG-13-005.

[5] Martin, Stephen P.: A Supersymmetry primer, hep$\mathrm{ph} / 9709356$.

[6] Aitchison, Ian J.R.: Supersymmetry and the MSSM: An Elementary introduction, hep-ph/0505105.

[7] CMS Collaboration: Search for a light charged Higgs boson in top quark decays in pp collisions at $\sqrt{s}=7$ TeV, JHEP 1207 (2012) 143.

[8] CMS Collaboration: Search for a Higgs boson produced in association with $b$ quarks and decaying into a b-quark pair in proton-proton collisions at $7 \mathrm{TeV}$, Phys. Lett. B 722 (2013) 207.

[9] CMS Collaboration: Search for SuperSymmetric Higgs boson states decaying into bb and produced in association with b-quarks in events collected by semi-leptonic triggers in pp collisions at $\sqrt{s}=7 \mathrm{TeV}$, CMS PAS HIG-12-027.

[10] CMS Collaboration: Search for MSSM Neutral Higgs Bosons Decaying to Tau Pairs in pp Collisions, CMS PAS HIG-12-050.

[11] CMS Collaboration: Search for Neutral MSSM Higgs Bosons in the $\mu^{+} \mu^{-}$final state with the CMS experiment in pp Collisions at $\sqrt{s}=7 \mathrm{TeV}$, CMS PAS HIG-12-011.

[12] J. E. Kim and H. P. Nilles: The $\mu$-problem and the strong CP-problem, Phys. Lett. B 138 (1984) 150.

[13] CMS Collaboration: Search for a light pseudoscalar Higgs boson in the dimuon decay channel in pp collisions at $\sqrt{s}=7 \mathrm{TeV}$, Phys. Rev. Lett. 109 (2012) 121801.

[14] CMS Collaboration: Search for a non-standardmodel Higgs boson decaying to a pair of new light bosons in four-muon final states, arXiv:1210.7619 [hep-ex].

[15] CMS Collaboration: Higgs to gamma gamma, Fermiophobic, CMS-PAS-HIG-12-022.

[16] CMS Collaboration: Search for the standard model Higgs boson in the $\mathrm{Z}$ boson plus a photon channel in pp collisions at $\sqrt{s}=7$ and $8 \mathrm{TeV}$, CMS-PAS-HIG13-006. 